\title{
«ESTADOS MÓRBIDOS» UN ANÁLISIS NECROPOLÍTICO DE LA FIBROMIALGIA EN LAS SOCIEDADES CONTEMPORÁNEAS
}

\author{
«Morbid States». A Necropolitical Analysis of \\ Fibromyalgia in Contemporary Societies \\ Dresda Emma Méndez de la Brena \\ Universidad de Granada \\ dresdaemma@gmail.com
}

\section{Resumen:}

Cada sociedad tiene sus propias enfermedades particulares y esas enfermedades dicen la verdad de su sociedad. El escenario que vivimos actualmente ubica la vida, cualquier vida, "entre la explosión y el estallido" (López Petit, 2015), donde no hay distinción entre vida y muerte sino debilidad como un continuo. Entre la explosión y el estallido hay cuerpos exhaustos. Cuerpos que muestran no sólo los aspectos debilitantes del neoliberalismo sino las economías que lo sustentan. Este artículo introduce el concepto de «Estados mórbidos» como una doble condición de vida que incorpora, por un lado, los regímenes afectivos contemporáneos de autoprecariedad y autorresponsabilidad que sitúan la enfermedad como un estado individual («estados mórbidos» en minúsculas). Por otro lado, («Estados Mórbidos» en mayúscula) el concepto se refiere a la necroadministración y aprovechamiento de la morbilidad a través de estructuras estatales que Ilamo «morbopolíticas», que posibilitan la producción de la propia enfermedad. En este círculo concéntrico, la fibromialgia se introduce como una enfermedad de género que se ha convertido en la fuente última para la extracción de vitalidad.

\section{Palabras Clave:}

Necropolítica, fibromialgia, biopoder, género.

\begin{abstract}
:
Each society has its own particular diseases, and those diseases speak the truth of each society. The scenario we are living today locates life, any life, "between the explosion and the outbreak" (López Petit, 2015), where there is no distinction between life and death, but instead there is debility as a continuum. Between the explosion and the outbreak there are bodies in exhaustion. Bodies that show not only the debilitating aspects of neoliberalism but the economies that sustain it. This article introduces the concept of «Morbid States» as a twofold condition of living that incorporates, on the one hand, contemporary affective regimes of self-precariousness and
\end{abstract}


self-responsibility that situate disease as an individual state («morbid states» in lower letters); on the other hand, the concept («Morbid States» in capital letters) refers to the necro-administration and use of morbidity through State structures I call «morbopolitics», which enable the production of the disease itself. In this concentric circle, fibromyalgia is introduced as a gendered disease that has become the ultimate source for the extraction of human vitality.

\section{Keywords:}

Necropolytics, Fibromyalgia, Biopower, Gender.

Recibido: 22/03/2021

Aceptado: 01/07/2021

El creciente incremento de los Síndromes de Sensibilización Central (SSC) que incluyen la Encefalomielitis Miálgica/Síndrome de Fatiga Crónica (enfermedad inmunológica y viral), las Sensibilidades Químicas Múltiples, la Fibromialgia (enfermedad neurológica y bioquímica) y la Sensibilidad Electromagnética no son sólo manifestación del alarmante peligro que supone el daño de los tóxicos al medio ambiente, el cambio climático o la alimentación transgénica sino que también son consecuencia de las injusticias y exclusión de las estructuras de la necropolítica neoliberal. Empezar con esta afirmación es, quizá, un reto ambicioso, porque para para entender los síntomas de las enfermedades contemporáneas, y en específico la fibromialgia, es necesario ir más allá del cuerpo con dolor y, ponerlo en conversación con estructuras que caracterizan al sistema neoliberal: productividad, eficiencia, y temporalidad. En otras palabras, empezar con esta afirmación es proponer el padecimiento de estas enfermedades más allá del cuerpo individual y entenderlas como nuevas gramáticas de malestar corporal inextricablemente ligadas a la violencia de las prácticas capitalistas neoliberales, sus estructuras de poder y de género.

El filósofo y escritor catalán, que padece Síndrome de Fatiga Crónica, Santiago López Petit (2014) menciona que, para entender la fenomenología propia de nuestro tiempo, es decir, la morbilidad invisible, es necesario concebir la progresiva identificación y alianza entre el capitalismo y la realidad de la época global. Para el autor, el malestar del cuerpo, que agobia a gran parte de la población, está relacionado con las enfermedades de la realidad (dolor cronificado, fatiga crónica, ansiedad), que asegura, son resultado de un "querer vivir», ante las lógicas de precarización extrema (Petit, 2014). Este artículo refiere precisamente al impacto de la realidad de la época global sobre los cuerpos, que no es otra cosa que estados de morbilidad precarizada. La precariedad que, como concepto y como condición ontológica, se extiende en diferentes contextos institucionales, gubernamentales y corporales requiere de un nuevo acercamiento para entender las condiciones que producen la morbilidad. Es por ello, que me pareció necesario traer el análisis de la necropolítica al estudio de la fibromialgia, y mirarla desde el sentir, pensar y hacer de las propias personas que padecen esta condición y que son afectadas por las estructuras que la habilitan. 
En este artículo presento un concepto de acuñe propio al que denomino como «Estados Mórbidos», y el cual trata de explicar el encuadre teórico contemporáneo que entrelaza la enfermedad y la necropolítica en ciertas poblaciones las cuales están situadas en un ensamblaje perverso de capacidad, debilidad y morbilidad que lleva a un proceso de «auto-precarización» (Lorey, 2016); es decir, un estado de enfermedad constante que se presenta como un problema individual y no como lo que es: resultado del neoliberalismo. «Estados Mórbidos», por tanto, es una herramienta teórica que me sirve para enmarcar la experiencia de enfermedades cronificadas como parte de los efectos de las estructuras morbopolíticas que distinguen al sistema neoliberal como son: la productividad, eficiencia y temporalidad y, que tienen como objetivo, la extracción de la vitalidad corporal que incrementa las enfermedades, en específico, en las mujeres.

«Estados Mórbidos» proviene de una genealogía que intersecta con el planteamiento de la teórica cultural Jasbir K. Puar $(2009,2011,2017)$ y la teórica de la discapacidad Margrit Shildrick (2015) sobre el concepto de «debilidad»; con el concepto de «necro-administración» de la filósofa mexicana Sayak Valencia (2010a), y con las reflexiones teórico-corporales de Santiago López Petit (2014) y Clara Valverde Gefaell (2015). Partiendo de las nociones foucaultianas de «biopoder» (Foucault, 1998 [1977]) y de las reflexiones del teórico poscolonial Achille Mbembe (2011) sobre «necropoder», «Estados Mórbidos» formula que la morbilidad (como condición ontológica de ciertas poblaciones) y la producción de la discapacidad (como extensión ontológica del derecho a dañar), son un ensamblaje contemporáneo destinado a la extracción de la vitalidad y de la obtención de plusvalía de cuerpos que, de otra manera, serían desechables. Personas con enfermedades crónicas, con discapacidad o con enfermedades mentales y demás grupos excluidos y precarios (Valverde, 2015) son parte de las poblaciones situadas en este ensamblaje perverso que intersecta la debilidad, morbilidad y discapacidad.

Para situar esta propuesta, propongo un recorrido teórico que se detiene en varias instancias. Comienzo exponiendo los conceptos teóricos que han ayudado a consolidar el concepto de «Estados Mórbidos». Con esto, intento aproximarme al reconocimiento de la importancia de un análisis necropolítico y de género, ya que permiten entender la serie de morbo-políticas dirigidas a las mujeres.

\section{HACIA LA MORBILIDAD: DE LA BIOPOLÍTICA A LA NECROPOLÍTICA}

Para entender cómo el concepto de Estados Mórbidos engloba varias técnicas del poder (productividad, eficiencia y temporalidad) que promueven la morbilidad contemporánea es necesario recurrir al concepto filosófico-político que refiere a una modalidad del poder específico que «hace vivir» en vez de «hacer morir»: «el biopoder». El concepto de biopoder desarrollado por Michel Foucault en la década de los setenta ha abierto el paradigma explicativo del poder que hace viable situar las condiciones materiales de posibilidad que demarcan el derecho de muerte y poder sobre la vida. En La voluntad de saber Foucault (1998[1977]) introduce un poder que distingue del derecho de muerte encarnado por el poder soberano. Este poder, según Foucault, está constituido por un cambio en la concepción de la gestión de poder que va más allá de la vida disciplinaria y punitiva que caracterizaba a los gobiernos de la 
Ilustración. En complementariedad del poder punitivo, existe una gestión estatal en donde la vida no necesariamente muere; en donde el poder se hace cargo de la vida.

Esta estatalización de lo biológico apunta a gestionar la población a través de dispositivos de vigilancia y control que aseguran salvaguardar y regular la vida de algunos mientras se excluye cruelmente a poblaciones no deseadas. Este cambio moderno incorpora un movimiento histórico del derecho de "hacer morir o de dejar vivir" al "poder de hacer vivir o de rechazar hacia la muerte" (Foucault, 1998:83); es decir, un cambio de una violencia directa y rápida hacia una sutil y más controlada forma de poder "que se ejerce positivamente sobre la vida, que procura administrarla, aumentarla, multiplicarla, ejercer sobre ella controles precisos y regulaciones generales" (Foucault, 1998:82). Concretamente, hablamos de un poder que entrelaza dos gestiones de la vida. La primera, centrada en anatomo-políticas que tienen como objetivo el sostenimiento del "cuerpo-máquina»; es decir, el cuerpo sostenido por sistemas de control eficaces (ej. educación) y económicos (ej. trabajo) que garantizan su correcta inserción en el sistema. La segunda, centrada en el "cuerpo-especie», enfocada al soporte de los procesos biológicos: nacimientos y la mortalidad, nivel de salud, la duración de la vida y la longevidad, etc. (Foucault, 1998). Estas políticas toman a su cargo una serie regulaciones de la población para organizar el poder sobre la vida: "un poder cuya más alta función no es ya matar sino invadir la vida enteramente" (Foucault, 1998:83). El biopoder sería entonces estudiado por Foucault, como una forma particular de poder que lleva al individuo a auto-constituirse como sujeto, pues la vida servirá de objeto y de modelo a las técnicas biopolíticas, pero al mismo tiempo, la vida será una posibilidad para pensar otras formas de poder y saber.

Para Foucault, la vida es el entramado entre las formas de poder y saber; sin embargo, en ninguno de sus textos parece precisar una definición del concepto de vida como estatuto ontológico propio. Quizá por ello, el filósofo Giorgio Agamben problematizará la «nuda vida» (zoé), como «simple hecho de vivir». Para dar respuesta a esto, en su libro Homo Sacer, Agamben (1995) estudiará la política sobre la vida a través de la aplicación de su análisis en los campos de concentración. El autor rechaza la tesis foucaultiana de que el aumento histórico del biopoder marcó el umbral de la modernidad. En cambio, afirma que el biopoder y la soberanía están fundamentalmente integrados, en la medida en que para el Estado moderno no es tan relevante la integración de la vida biológica en la esfera de la política, sino que el poder moderno del Estado saca a la luz el nexo entre soberanía y cuerpo biopolítico. Por tanto, lo que los campos de concentración revelaron fue el paradigma totalitario de gestión estatal de la vida. Una vida que ha sido separada de su forma, y que puede ser asesinada pero no sacrificada. Una vida que ha sido expuesta, una «vida desnuda», a la que no se le está «haciendo morir», como argumentaría Foucault, sino en la que los individuos son completamente despojados de todo valor político, y con ello, objetos de una violencia que excede la esfera del derecho en el contexto totalitario.

Agamben relaciona la vida desnuda con el poder soberano, ya que este último se establece a través de la producción de un orden político basado en la exclusión de la vida desnuda. Esto se logra mediante la promulgación de un estado de excepción en donde la vida humana está dentro de una lógica binaria de exclusión-inclusión, una vida incluida dentro del orden jurídico, pero a partir de su exclusión; es decir, de su 
capacidad de ser asesinada. La soberanía que desarrolla Agamben se caracteriza por la separación, por la cesura, por la suspensión de la vida para capturarla. Esto es importante, ya que como veremos más adelante, el Estado Mórbido funcionaría también con la misma lógica: inclusión de la excepción, es decir, un vacío o espacio de indiferenciación, en donde la vida mórbida puede ser asesinada sin ser un asesinato; en donde la persona enferma puede ser dejada para vivir una muerte en vida.

A pesar de la complementariedad que Agamben aportó al análisis de Foucault, ninguno de estos autores profundizará en el papel de la raza y el racismo como el despliegue y manifestación sobre la vida como lo hará el teórico poscolonial Achille Mbembe. En Genealogía del Racismo (2006), libro resultado del curso en el Collège de France, Foucault analiza las teorías raciales ofreciendo una descripción genealógica de las formas en que las formas modernas y biológicas de racismo surgieron a finales del siglo XIX. Sin embargo, fue Achille Mbembe quien aportó una lectura fundamental para comprender cómo en la economía del biopoder, "la función del racismo consiste en regular la distribución de la muerte y en hacer posibles las funciones mortíferas del Estado" (Mbembe, 2011:23). A diferencia de Agamben, Mbembe sitúa su teoría dentro del relato histórico de la aplicación del poder del Estado a la esclavitud y el colonialismo como proceso y temporalidad en donde convergen las primeras manifestaciones de la experimentación biopolítica; específicamente, en la administración de las plantaciones.

Los campos de cultivo de plátano son, para Mbembe, la representación del terror colonial en donde existe una "sumisión de la vida al poder de la muerte (política de la muerte)" (Mbembe, 2011:74); de aquí, la noción de «necropolítica». La propia estructura del sistema de plantación permitía la implantación del terror, en donde la vida del esclavo estaba a la especulación y voluntad del amo. Dice Mbembe "El esclavo es, por tanto, mantenido con vida, pero mutilado en un mundo espectral de horror, crueldad y desacralización intensos" (Mbembe, 2011:33). De manera tal, que la vida del esclavo se convertía en una forma de «muerte-en-la-vida» (Mbembe, 2011) y, la violencia extrema, el modo operandi de esta relación. La particularidad y, por tanto, perversidad de este sistema es que las formas de violencia no involucran el asesinato directo de individuos sino su lenta degradación biológica o un estado permanente de lesión (Mbembe, 2011).

Vemos como Mbembe va un poco más allá de la idea foucaultiana del derecho a matar al derecho de exponer a las personas a la posibilidad de muerte; no para hacer que alguien muera inmediatamente sino para dejarlos morir. En el trabajo de Mbembe, los cuerpos colonizados se mantuvieron en un estado de lesión gradual a través de procesos de dominación colonial al ser considerados cuerpos desechables y, que pueden, por tanto, sufrir el desgaste. Esta herida permanente encaja con la experiencia social que degrada permanentemente a poblaciones distribuidas en contornos cambiantes de raza, clase, discapacidad y género en diferentes contextos neoliberales actuales. Estos grupos poblacionales, viven sin muerte inmediata o deliberada, pero a través de una lenta exposición a la violencia y sistemas de opresión, se mantienen marginadas en situaciones y espacios de subyugación. 
Un ejemplo de las políticas de degradación de la vida en un contexto neoliberal actual es ilustrado por la teórica cultural, Lauren Berlant. La autora argumenta que la salud de poblaciones enteras puede ser erosionada gradualmente a través de las maquinaciones del neoliberalismo. Para Berlant (2007), la epidemia de obesidad en los Estados Unidos es una forma de entender la destrucción de la vida y de los cuerpos en zonas de lo ordinario bajo el régimen contemporáneo del capital. La obesidad, señala Berlant, no debe analizarse solamente como práctica individual sino un conglomerado de circunstancias encarnadas (ligadas a la clase, la raza, la edad, el género) $y$, de condiciones estructurales enmarcadas en políticas corporativas y gubernamentales (el estancamiento de la clase media, jornadas de trabajo más largas, contratos parciales, ahorro de tiempo en comida inmediata), que acompañan cada acto de comer. Berlant presenta el concepto de "muerte lenta», no necesariamente aislado de actos de brutalidad obvia del capitalismo en los espacios de producción y en el resto de espacios vitales en términos de Mbembe o Agamben, pero si oculto en formas de daño a largo plazo, cuyas cualidades y contornos en el tiempo y el espacio

are often identified with the presentness of ordinariness itself, that domain of living on, in which everyday activity; memory, needs, and desires; diverse temporalities and horizons of the taken-for-granted are brought into proximity $^{1}$ (Berlant, 2007: 754).

La muerte lenta ocurre en la temporalidad de lo ordinario, no dentro de temporalidades de emergencia o excepción. La muerte lenta tiene su propia temporalidad en donde "the structural inequalities are dispersed, the pacing of their experience intermittent, often in phenomena not prone to capture by a consciousness organized by archives of memorable impact" [las desigualdades estructurales se dispersan, el ritmo de su experiencia es intermitente, a menudo en fenómenos no propensos a ser capturados por una conciencia organizada por archivos de impacto memorable] (Berlant, 2007:279). En otras palabras, el concepto de muerte lenta describe las maquinaciones del neoliberalismo dirigidas a regular los actos más ordinarios del ser hacia una morbilidad corporal ( $y / o$ mental) constante.

De tal lectura, podemos apreciar como el cuerpo ha sido el blanco fundamental tanto de la biopolítica como de la necropolítica, y como se vuelve objeto de opresión, eliminación, silenciamiento, reemplazo, extracción, consumo. Sin embargo, hay una tercera lectura del valor de la vida dentro de la propuesta del Estado Mórbido y, que tiene sus fundamentos en la muerte lenta, esto es, la máxima rentabilidad de la enfermedad, del cansancio, del desgaste en un escenario necropolítico; en otras palabras, en la morbilidad. La morbilidad es parte del ensamblaje necropolítico dentro de un régimen global y nacional de políticas dirigidas a la atenuación física controlada

\footnotetext{
${ }^{1}$ Las citas no son traducciones profesionales. He traducido las mismas directamente desde el texto en inglés, texto incluido en el cuerpo del texto cuando es de menos de 40 palabras y como notas al pie cuando rebasa esta cantidad para que él o la lectora puedan referirse a la obra original.

[se identifican a menudo con el presentismo de lo ordinario, ese dominio del vivir en el que la actividad cotidiana; memoria, necesidades y deseos; diversas temporalidades y horizontes de lo que se da por sentado cuando son puestos en proximidad] (Berlant, 2007: 754).
} 
de los cuerpos. Esto parece particularmente aplicable a personas con Síndrome de Sensibilización Central (SSC) que, bajo la política de desgaste de cuerpos no rentables, forman una comunidad que está «muerta en vida» (Valverde, 2015). Al igual que la pandemia de la obesidad que refiere Berlant, las maquinaciones del neoliberalismo dirigidas a las personas enfermas de SSC, se articulan también de forma lenta, paulatina y consistente, constituida por engranajes perversos para administrar y rentabilizar el estatus de muerte-en-vida. ¿Con qué fin? Para contestar esta pregunta es necesario incorporar a la conversación la cuestión de la debilidad, especialmente en su relación con la discapacidad y la necropolítica en el marco del régimen neoliberal.

\section{"ESTADOS MÓRBIDOS": LA MORBILIDAD COMO POLÍTICA «GORE»}

Hasta ahora, he tratado de establecer una genealogía del desarrollo constitutivo del binomio bio-necropolítico que ha favorecido el control de la vida y la muerte, así como la constitución de prácticas materiales y discursivas destinadas a la sujeción de los individuos. En este paradigma en donde vida y muerte son gestionadas, manipuladas y rentabilizadas para el sostenimiento del poder soberano, se incorporan otros sistemas de desgaste y degradación, en donde la muerte ya no se concibe en términos absolutos sino de forma paulatina y lenta. Es precisamente en este estado de desgastamiento donde sitúo el concepto de Estado Mórbido.

La morbilidad entendida como una condición socio-ontológica de vida, esto quiere decir, un acercamiento a la morbilidad como relacional más que individual, debe ser puesta en conversación con el «corpus» político que la genera, sostiene y mantiene. Conversación que está en dialogo con los «saberes sujetos» (Foucault, 2006), «saberes subalternos» (Medina-Doménech \& Rosón, 2017) o quizá en esta sintonía de saberes, con los «saberes tullidos» (Platero, 2014) de los cuerpos con morbilidad. Saberes cuyo contenido ha sido invisibilizado y descalificado por bloques de saber normalizados y jerárquicos que se posicionan por encima de ellos al considerarlos "saberes ingenuos, jerárquicamente inferiores, por debajo del nivel de conocimiento o cientificidad requerido" (Foucault, 2006:18). Por ello, en este capítulo, y a lo largo de este trabajo de investigación, construyo teoría junto con saberes encarnados que me permiten dialogar desde una posición diferencial la construcción del conocimiento.

El concepto de Estados Mórbidos proviene de la lectura que realizo del trabajo narrativo y encarnado de dolor y fatiga crónica de Santiago López Petit y Clara Valverde y de las luchas y memoria de los cuerpos de mujeres con dolor cronificado que, como menciona López Pétit "vive[n] entre la implosión y el estallido" (2014:75). El autor utiliza la dureza poética de esta frase para referirse a su posición desde la vida fatigada que es su cuerpo. Un cuerpo con Síndrome de Fatiga Crónica-Encefalomielitis Miálgica (SFC-EM), que como su nombre lo indica, es una fatiga intensa y dolor cronificado generalizado. El lugar que habita el cuerpo de López Petit es un espacio muy específico, que coincide con el de 160.000 personas afectadas de fatiga crónica, cerca de 38.000 de síndrome de fatiga crónica y 7.500 con sensibilidad química múltiple en España (Ajuntament de Terrassa, s. f.). Su cuerpo que adolece de fatiga crónica no se refiere solamente a la condición de su enfermedad, ni tampoco al mero cansancio de su cuerpo. Para Petit, entre la implosión y el estadillo hay todo un 
sistema que se sostiene del vacío, o más bien, de los cuerpos en vacío, cuerpos desgastados por vivir la enfermedad en forma de activismo permanente. Cuerpos con una militancia en dedicación intensa de soportar y gestionar mínimamente una vida que pueda insertarse en la productividad de la normalidad. Un sistema que permite reintroducir la fatiga para seguir extrayendo algo de ella. La fatiga, la debilidad y la morbilidad al servicio de la acumulación capitalista.

Entre la implosión y el estallido me sirve como analogía poética para situar la morbilidad en conversación con al acercamiento académico que Jasbir Puar $(2009,2017)$ hace de las políticas espacio-temporales que simultáneamente actúan con regímenes de muerte mientras permanecen ligadas a la optimización de la vida. Entre la implosión y el estallido, no hay distinción entre la vida y la muerte, sino un cuerpo en extenuación, en debilitamiento. Entre la implosión y el estallido hay un estado basado en el sostenimiento de la enfermedad. El cuerpo que ocupa ese espacio, es un cuerpo que está sujeto a los debilitantes aspectos del neoliberalismo y las economías que rodean su debilidad (Puar, 2017); es decir, un sistema extractivo (Gómez-Barris, 2017) que sitúa la vida en deuda permanente.

Autoras como Margrit Shildrick (2015) y la ya citada Jasbir Puar $(2009,2017)$ han trabajado en la incorporación de los estados de debilidad, discapacidad y los afectos en los Estudios Críticos de la Discapacidad. En la argumentación de Puar (2017), la debilidad no es, sola o exclusivamente, un estando ontológico de deficiencia en alguna capacidad sino una herramienta política que puede ser aplicada para analizar los costes que algunos cuerpos pueden llegar a pagar por el progreso. La importancia del análisis de Puar es que desplaza la discapacidad no como una identidad meramente oprimida de los cuerpos con discapacidad sino, en cambio, la considera como parte de un ensamblaje bio-necro-político neoliberal que implica y permea a todos los sujetos encarnados.

Because there are gradations of capacity and debility in control societiesrather than the self- other production of being/not being - the distinction between disabled and non-disabled becomes fuzzier and blurrier $^{2}$ (Puar, 2017:22)

Como tal, ya no existe un organismo que cumpla con el estándar de personas con capacidades adecuadas, sino solo gradaciones de capacidad y debilidad que desdibujan la distinción entre la discapacidad y no discapacidad. Con esta gradación, Puar quiere ir más allá del binomio bio-necropolítico, utilizando la debilidad y la capacidad como elementos que confunden las distinciones entre vivir y morir, reflejando los parámetros contradictorios de la bio y necro política. Continuando con Puar (2017), la autora argumenta que todos los cuerpos dentro del capitalismo neoliberal están "being evaluated in relation to their success or failure in terms of health, wealth, progressive productivity, upward mobility, [and] enhanced capacity" [siendo evaluados en relación con su éxito o fracaso en términos de salud, riqueza, productividad progresiva, movilidad ascendente, y capacidad mejorada] (Puar,

${ }^{2}$ [Debido a que hay gradaciones de capacidad y debilidad en las sociedades de control-, en lugar de la producción del yo-otro de los procesos del ser/no ser, la distinción entre discapacitados y no discapacitados se vuelve cada vez más difusa] (Puar, 2017: 22). 
2017:155) y, esto es, porque dados los desarrollos biopolíticos en el capitalismo neoliberal, la normalización del cuerpo discapacitado ya no es el foco principal de la intervención médica. Quizá López Petit pueda ayudar a ejemplificar esta última parte.

En el capitalismo neoliberal, los cuerpos ya no son valuados sólo por su capacidad de trabajo sino también por la información que producen (Puar, 2009). A partir de lo que López Petit (2017) denomina «poder terapéutico», se intenta rehabilitar, no tanto curar, al cuerpo enfermo, creando así, una economía de debilidad la cual sirve a los intereses del capitalismo neoliberal mientras que reforma la construcción o ensamblaje de la morbilidad. Menciona Petit,

El poder terapéutico cumple tres funciones claves: el poder terapéutico nos mantiene con el mínimo de vida necesaria para poder soportar y no caer fuera de la movilización. En el poder terapéutico hace posible el ser precario que se nos clava; hace posible el ser precario que persiste. En ese sentido, el poder terapéutico es antes que medicamentalización generalizada. La gestión psicológica, psiquiátrica, medica de nuestra vulnerabilidad. (Petit, 2017, min. 31:08 - 32:00)

Lo que sostiene al capitalismo, por tanto, es la vida misma, y en este punto, cualquier vida, inclusive la mórbida, mientras esté al servicio de la acumulación capitalista. Sin embargo, más allá del paradigma biopolítico de control de la vida para la productividad, nos encontramos con una sociedad que avanza por un paradigma distinto, ya no basado en el exceso de productividad sino en el exceso de positivización (el afán de rendimiento, de felicidad, de estar mejor), que desplaza el malestar social al cuerpo individual, configurando distintas formas de enfermedad.

En el libro La Sociedad del Cansancio, Byung-Chul Han analiza lo que denomina como "sociedad de rendimiento", que se caracteriza por el "verbo modal positivo poder" (2012 [2010]: 26). Este poder sustituye el paradigma disciplinario, al que se refiriera Foucault décadas atrás, por un modelo positivo o psicopolítico de hacer, a partir de incentivos dirigidos a una versión ficticia de libertad, felicidad y a un exceso de positividad. En la sociedad de rendimiento, ya no se insiste en la prohibición, disciplinamiento y el mandato externo en el ámbito laboral, sino en la capacidad de iniciativa y la promoción.

Con el fin de aumentar la productividad se sustituye el paradigma disciplinario por el de rendimiento, por el esquema positivo del poder hacer, pues a partir de un nivel determinado de producción, la negatividad de la prohibición tiene un efecto bloqueante e impide un crecimiento ulterior. La positividad del poder es mucho más eficiente que la negatividad del deber (Han, 2012 [2010]: 27).

La psicopolítica para Han es la nueva estrategia del poder del neoliberalismo, porque la «psique», es la nueva fuerza productiva. Ya no es el cuerpo el principal protagonista de la sociedad disciplinaria biopolítica, sino la optimización de los procesos psíquicos y mentales; es decir, formas más refinadas de explotación. El concepto de Estados Mórbidos reconoce la psicopolítica como parte del vocabulario existente para nombrar formas de gestión de vidas invivibles. Sin embargo, el análisis de Han deja a un lado toda implicación del género como técnica disciplinaria que hace que unos cuerpos se cansen más que otros; que unos cuerpos se enfermen más 
que otros. Si, "en esta sociedad de obligación, cada cual lleva consigo su campo de trabajos forzados" (Han, 2012 [2010]: 48), los cuerpos de las mujeres Ilevan en su cuerpo un cansancio, un debilitamiento, un malestar por un nivel de productividad obtenida por la técnica disciplinaria del género; esto es, por el imperativo del deberser mujer que produce un cansancio y un agotamiento excesivos. Si toda época tiene sus enfermedades emblemáticas, vivimos en tiempo donde la depresión, la ansiedad, la anorexia, las enfermedades de los sistemas inmunológicos como el síndrome de fatiga crónica, la sensibilización química múltiple, la fibromialgia son enfermedades que llevan la marca de género.Además, es evidente renuncia al reconocimiento de la genealogía feminista de donde ha desarrollado su concepto. «El capitalismo de la emoción» como Han lo llama es no otra cosa que lo que ya han desarrollado feministas dentro del área de los Estudios Culturales sobre las implicaciones de la emocionalización o circulación afectiva (ver Ahmed, 2004) en el proceso productivo dentro del marco neoliberal. Por ello, prefiero recuperar el trabajo de Margrit Shildrick (2015) y Sara Ahmed (2019) que me proporcionan argumentos más interesantes que sitúan cómo la debilidad juega un papel importante, cuando se le añade, además, una estrategia en forma de afecto, de esperanza de «seguir viviendo», de promesa de «estar mejor» desde un análisis feminista y de género.

Por tanto, en el análisis de Ahmed, la promesa es una técnica afectiva de autoregulación neoliberal. Como ya lo venía desarrollado en su libro La política cultural de las emociones (2015[2004]), existe una articulación cultural de la sociedad alrededor de las emociones, en donde éstas juegan un papel fundamental en las estructuras de poder al reconfigurar constantemente la organización social. En su libro La promesa de la felicidad (2019), la autora profundiza aún más en las emociones centrándose en la felicidad como una técnica diseñada para vivir bien y asociada a la determinación, valoración y clasificación de determinados tipos de vida sobre otros. Por tanto, hay una direccionalidad hacia la gestión de la felicidad, de la mano de los discursos positivistas del neoliberalismo, en donde "la felicidad dicta la organización del mundo" (Ahmed, 2019: 22).

Lo que caracteriza a la sociedad contemporánea occidental, argumenta, es una serie de promesas con el fin de lograr el buen vivir, mediante la garantía de la felicidad. Para Ahmed, la promesa, "es una garantía, una declaración positiva que tiene la intención de ganar credibilidad y confianza en el cumplimiento de una expectativa" (Ahmed, 2019: 73-74). A la promesa entonces la acompaña una manipulación del deseo, es decir, el convencimiento de que el "sentirse mejor es mejorar" (Ahmed, 2019: 32; itálicas en original), aunque sea a costa de nuestra propia debilidad. La promesa se convierte en gestoría de nuestros deseos, de los espacios que ocupan y de los cuerpos que lo habitan. Se trata, en consecuencia, de la promesa como disciplina, direccionamiento u orientación, reforzado por una serie de técnicas ideológicas (e.g. psicología positiva, libros de auto-ayuda, etc.) destinadas a la propia superación, poniendo el énfasis en el logro personal como medio para alcanzar la felicidad. Menciona Ahmed, "De esta forma, la felicidad no solo se convierte en una responsabilidad individual, una reformulación de la vida como proyecto, sino también en un instrumento; es decir un medio para un fin, y no sólo un fin en sí mismo" (Ahmed, 2019: 34). 
De suerte tal que, la promesa es utilizada como una técnica estatalizada para el control poblacional a través de la auto-regulación para canalizarnos por la buena senda (la del sujeto neoliberal) y, la felicidad, el instrumento afectivo que garantiza la autogestión de nuestra infelicidad. La promesa de recuperación es, entonces, una técnica ilusoria que da sustento a nuestras expectativas sobre el porvenir. Promesa que deseamos, aun cuando sea una estrategia de responsabilidad personal para solventar la propia debilidad. Aquí podemos ver conexiones con lo que la teórica cultural Lauren Berlant (2011) denomina como «optimismo cruel»; es decir, el deseo o la creencia equivocada de que se conseguirá la felicidad personal a través del trabajo duro. Por tanto, para que la promesa de mejoría funcione ideológicamente, no así empíricamente, necesita de una violencia discreta que permita una erosión de la vitalidad del cuerpo que, sitúa tanto al vivir y morir, en una zona específica de proximidad y precariedad.

De esta manera se fusiona la promesa de mejoría con una movilidad social ficticia en donde estamos, "compelled to become consumers regardless of the risk of disappointment and debt illusion of getting better [obligados a convertirse en consumidores independientemente del riesgo de decepción y la ilusión de la deuda de mejoraría] (Shildrick, 2015: 6). A través de la gestión voluntaria de nuestros propios afectos, aseguramos un mínimo de vida necesaria, aunque precaria, para soportar la vida. Por ello, Puar sitúa dentro de los escenarios bio y necropolíticos, la esperanza como un dispositivo afectivo que genera debilidad mediante la extracción de fantasías de mejora. Al complejizar la idea de la autora Sarah Lochlann Jain (2007), Puar (2009) argumenta que vivimos en «tiempos de pronóstico», temporalidad que relaciona la vida con ideas sobre variabilidad y riesgo; es decir, el vivir (y morir) en relación con la probabilidad estadística de poblaciones frente a la salud, la enfermedad, la discapacidad, y la debilidad. Temporalidad sostenida por la política de la esperanza en donde la promesa de recuperación es trabajar la propia vida precaria para darle rentabilidad. Por tanto, tiempos de pronóstico es una temporalidad no de mejoría sino de mantenimiento.

La taxonomía de la violencia dentro del capitalismo que nos ofrece la filósofa transfeminista mexicana Sayak Valencia se añade a esta conversación como fundamental para explicar la producción de la enfermedad como una política afectiva «gore» en la sociedad actual. Para esta autora, nos encontramos en una intersección vital en donde la episteme de la violencia ha encontrado su nicho de expansión en un sistema que reboza en la economía hegemónica y global y en los espacios de extracción de la vitalidad productiva. En un análisis situado de la realidad fronteriza de donde proviene, su apuesta es nombrar procesos de espectaculización de la violencia que, aunque evidentes por su performance grotesco, parecieran perderse en su exceso de visibilidad. "Capitalismo Gore» es el término que escoge la autora para nombrar una serie de técnicas y prácticas de enarbolamiento de la violencia destinadas a hacer de la muerte y su especularización sinónimo de valor. La vida, ya no es importante, ahora la muerte es el campo de extracción de la vida productiva. Aunque su análisis se basa en la formación de subjetividades de violencia (sujetos endriagos) que se producen en contextos liminales de violencia (frontera); la producción del valor y de la vida en la sociedad actual, dependen de una sustitución de la fuerza de trabajo, por medio de, "prácticas gore, entendidas como el ejercicio 
sistemático y repetido de la violencia más explícita para producir capital" (Valencia, 2010a, p. 51). Necroempoderamiento y necroprácticas tránsfugas y distópicas (asesinatos, mutilamientos, secuestros) son herramientas gore que la autora nombra y que dan acceso a los beneficios de una economía de la muerte. Así, la visión que propone Valencia sobre capitalismo gore explica un fenómeno en donde la violencia estructural es cómplice y benefactora del «necro-capitalismo» (Tyner, 2019).

Ahora bien, identifico una conexión fundamental entre el capitalismo gore del que habla Valencia, los regímenes afectivos que menciona Shildrick y Ahmed y el concepto de Estado Mórbido que propongo. Esta conexión radica en las estructuras perversas del Estado capitalista para extraer ganancias a partir de la configuración de técnicas afectivas rizomáticas que se expanden en el mundo de los deseos. Los procesos de enfermedad son, por tanto, resultado de las estructuras violentas del Estado Mórbido que, a través de modulaciones afectivas,

transforman contextos y/o situaciones de vulnerabilidad y/o subalternidad en posibilidad de acción y autopoder, pero que los reconfiguran desde prácticas distópicas y autoafirmación perversa lograda por medio de prácticas violentas que son rentabilizables dentro de las lógicas de la economía capitalista (Valencia, 2010b, párr. 4).

En el Estado Mórbido, el necro-empoderamiento se constituye por las prácticas de habilitación de nuestro propio desgaste. La morbilidad como política gore es la implantación de discursos neoliberales ya no de forma directa o expuesta, sino por el contrario, en una especie de posibilidad de acción ficticia en donde somos responsables de administrar nuestra propia debilidad. Tenemos entonces, por un lado, «estados mórbidos» (minúscula) incorpora regímenes afectivos contemporáneos de auto-precarización y auto-responsabilización que definen la enfermedad como estado individual y de autogestión de la debilidad. Por otro lado, «Estados Mórbidos» (mayúscula) refiere a la necro-administración y aprovechamiento de la morbilidad a través estructuras del Estado como la productividad, eficiencia y temporalidad que, en conjunto, habilitan la producción de la enfermedad. Ambos registros emplazan un círculo concéntrico del Estado capitalista neoliberal como productor de la morbilidad social y, en su perversidad, de la morbilidad individual. En otras palabras, la morbilidad como una experiencia particular de los cuerpos que es resultado de una inducción política hacia una mayor precariedad, debilidad, responsabilización y auto-gestión de quien la padece. Aquí, pongo en valor las reflexiones de Diego Sztulwark (2016) quien argumenta precisamente que una de las estrategias del neoliberalismo ha sido inculcarnos una «sensibilidad de desgaste» como acondicionamiento colectivizado, a través un lenguaje promisorio y un «sentido común» (Gago, 2015) entumecido de esto es lo que hay, y, si se quiere algo más, la responsabilidad es individual. La aceptación de esta realidad es lo que Sztulwark denomina "sensibilidad neoliberal», que podría entenderse para mis objetivos como una forma de gubermentabilidad (Foucault, 1998) que a través de un lenguaje neoliberal promisorio manipula o modula los afectos (Puar, 2017) hacia la resignación de la aplicación de las políticas neoliberales de explotación o debilitamiento a partir de un asentimiento pleno y sin cuestionamientos del malestar. 
El planteamiento de Sztulwark se cruza dialécticamente con el de la escritora mexicana Irmgard Emmelhainz (2016) quien, en su libro Tiranía del sentido común, realiza un análisis sobre cómo el neoliberalismo — en el contexto del Estado Mexicano-, se ha convertido en una forma de sentido común intrínsecamente enraizado en la vida, como sentido común, sensibilidad y afecto de los sujetos, dándole forma a la realidad que habitamos. Para la autora, el neoliberalismo es una "una sensibilidad que trabaja los deseos más íntimos, colonizando nuestros sueños, canibalizando nuestros ideales de libertad y regurgitándolos como estrategias de control social" (Emmelhainz, 2016: 40). La sensibilidad neoliberal utiliza palabras como «democracia», «desarrollo», «mejora», «seguridad», «eficiencia», "autonomía», y "auto-suficiencia», como "excusas para modelar nuevas formas de vida y de ganarse la vida que implican la reconversión y que van desde la autoexplotación, explotación, esclavitud, exclusión, endeudamiento, despojo, expropiación y hasta la muerte" (Emmelhainz, 2016: 72). En esta genealogía que abastece el enfoque y propuesta de Estado Mórbido, añado al análisis SztulwarkEmmelhainz, el impacto de la sensibilidad neoliberal como una estrategia políticoafectiva gore que habilita la enfermedad y nuestros estados mórbidos. La sensibilidad neoliberal nos convence sensualmente que la única manera de mejorar, de estar mejor, es trabajar más allá de nuestras capacidades mentales y físicas, es enfermar.

En el Estado Mórbido, la sensibilidad neoliberal actúa a partir de técnicas de violencia discreta como la promesa de la mejoría, el auto-empoderamiento, la autoayuda, la auto-superación, etc. Sin embargo, quiero aclarar que en ningún momento intento sugerir que la aceptación o la condonación de los efectos de la sensibilidad neoliberal, es este caso, a partir de la constitución de la propia enfermedad, es responsabilidad del individuo o, en su defecto, de los grupos marginalizados. Porque, parafraseando a López Petit (2014), ¿Quién no quiere hacer pactos malditos con la vida para no estallar y seguir viviendo? He aquí, la complejidad del Estado Mórbido. Sacar ventaja de la subjetivación sensible, de la debilidad para distribuir la vulnerabilidad sacando ventaja de su gestionamiento. A este acto de política estatal perversa y morbosa que se deleita en la habilitación de la enfermedad, la he denominado como «morbo-política». Con la propuesta de morbo-política nombro a todas las técnicas estatales que aplicadas a cuerpos que medicalizan su vulnerabilidad; cuerpos cuyo sentido de auto-realización es gestionarse la debilidad para no ser estigmatizados y, que, al hacerlo, su vulnerabilidad y debilidad es canalizada como condición vital extractiva de la cual el capitalismo no solo depende, sino que se beneficia. La morbopolítica como brazo asfixiante del Estado Mórbido, a partir de una sensibilidad neoliberal, convierte los procesos de auto-debilitamiento en cotidianidad. De modo tal, vivir y morir ya no son las dos políticas de control subyacentes de cualquier organismo. La morbilidad es, ahora, una nueva forma de política.

Con el concepto de Estado Mórbido me refiero, por tanto, a la exposición de las personas a la enfermedad; no para hacer que alguien muera inmediatamente sino la vida enferma como una nueva forma de acción directa a la despotenciación de la vida; a la expropiación de la fuerza vital humana para transformarla en fuerza de trabajo y fuente de acumulación de las sociedades capitalistas contemporáneas. En 
este nuevo pliegue necropolítico, la morbilidad es una nueva creación de formas de existencia que reverbera con sus efectos sobre la sociedad en su conjunto. La crisis actual del incremento de los Síndromes de Sensibilización Central es una oportunidad para ver los efectos de la morbo-política que insiste en que las enfermedades cronificadas deben tratarse como un hecho biológico y no un efecto políticoeconómico. Por tal motivo, el análisis de la fibromialgia es el mejor lugar para efectuar el análisis de los efectos del Estado Mórbido, no sólo porque la fibromialgia se presenta en un grupo poblacional amplio (de mayoría mujeres), sino porque sus efectos, precisamente por este motivo, no son sólo diferentes sino más perversos, porque la expropiación de la fuerza vital humana conlleva un análisis más complejo si se le añade la perspectiva de género.

\section{LA ENFERMEDAD DE LAS MUJERES: LA FIBROMIALGIA}

De acuerdo a la Sociedad Española de Reumatología (2001), la prevalencia en España es del $2,73 \%$ de la población general mayor de 20 años, según el estudio EPISER sobre Prevalencia e impacto de las enfermedades reumáticas en la población adulta española. La prevalencia en España, según datos de este estudio, se sitúa en un 2,4\%, con un claro predominio en mujeres $(4,2 \%$ frente a $0,2 \%$ en hombres) y un pico de prevalencia entre los 40 y los 49 años. Sólo en Andalucía se calcula que más de 300.000 personas sufren SSC (SESSEC. Más de 300.000, 2020). En cuanto a la distribución por grupos de edad, la prevalencia máxima aparece entre los 40 y los 49 años (4,9\%). Es más frecuente en mujeres (90\%), entre 20 y 55 años de edad.

Como menciona la activista feminista, poeta y disidente sexual argentina Valentina Stutzin (2019), la fibromialgia es un diagnóstico incómodo para I*s demás porque trae sospechas de credibilidad dado que su invisibilidad presupone a quienes la padecen como "débiles, manipuladoras, pusilánimes y/o exageradas" (p. 175). Alejado de la vista, la invisibilidad del sufrimiento y/o de la persona enferma se oculta de los espacios, se relativiza, se culpabiliza, se recrimina o trivializa. Valverde (2015) toma las reflexiones de la antropóloga Julienne Lipson Ilamando a esto "violencia discreta», en donde,

Primer[o], no se ve a los enfermos porque en la mayoría de los casos están encerrados en sus casas, demasiado enfermos para salir. Segund[o], si consiguen salir, no se les ve porque muchas enfermedades no tienen señales externas. La tercera forma de invisibilidad, según Lipson, es cuando la persona dice que está enferma pero no se la cree ni se la toma en serio (apud., Valverde, 2015, p. 30).

Esta situación de desigualdad y discriminación pone en evidencia las injusticias que viven las mujeres con fibromialgia. Mujeres con cuerpos exhaustos que cumplen doble jornada laboral basada en una división sexual del trabajo en casa, al paralelo de otra productiva, con precarización y salarios cada vez más pauperizados, con jornadas laborales más largas y en donde, en muchos casos, se sufre acoso laboral. Mujeres que deambulan en una suerte de «muerte lenta» (Berlant, 2007) en un peregrinaje médico buscando un diagnóstico correcto, mientras se vive en un suplicio al estar angustiadas al no saber qué les ocurre, y no poder explicar o ser creídas por sus familias. Mujeres sobremedicalizadas pero que son dejadas a la suerte de los 
«medicamentos huérfanos»; es decir, medicamentos que la industria farmacéutica tiene poco interés en desarrollar y comercializar por el elevado coste de llevar un medicamento al mercado del cual no se recuperaría con las ventas esperadas del producto (Campillo Artero y Peiro, 2009). Mujeres en peregrinaje jurídico en un intento de recibir apoyo por parte del Estado, ya que hacer frente a su condición es muy costoso porque se necesita de un reumatólogo, un psicólogo, un nutriólogo, entre otros especialistas. Mujeres que se sienten poco valoradas en sus entornos, mujeres explotadas por la condición de género construido sobre las demandas de amor, afecto y cuidado que recaen en ellas en el marco de las relaciones de pareja y filiales. Mujeres que sostienen durante años episodios de violencia machista. Mujeres cuya esperanza de vida es de 25 años menor que el de la población en general (Valverde, 2015).

Como vemos, los cuerpos de aquellas mujeres que viven con enfermedades marginadas, comparten una realidad que quienes se benefician del sistema, no se imaginan. Su cuerpo está siendo utilizado para perpetuar la necropolítica del neoliberalismo, a partir de un sistema de género diferenciador y discriminador que se encarga de extraer y controlar su potencial vital, sus tiempos, sus estados emocionales, sus procesos de mejoría. El cuerpo de las mujeres con Síndromes de Sensibilización Central, se ha trasladado a un "espacio intersticial» (Critchley apud Valverde, 2015), un espacio opaco, que no está ni fuera ni dentro del sistema. Es decir, excluidas de la sociedad porque no se les quiere ver, pero que, cuando su presencia se hace visible, pone en evidencia las injusticias y las estructuras de poder del Estado Mórbido (Valverde, 2015). Se observa en este encuadre de vulnerabilidad, el funcionamiento del Estado Mórbido conjugado con la opresión de género. El Estado Mórbido habilita discursos médicos, sociales y económicos sostenidos por la división sexual del trabajo y los mandatos de género para construir un «sentido común» o una "sensibilidad neoliberal» (Emmelhainz, 2014; Gago, 2015; Sztulwark, 2019), que utiliza la movilización de afectos positivos para encausar la extracción de la fuerza vital, y en el caso de las mujeres con fibromialgia, dirigirla hacia el sometimiento de su tiempo, de su productividad, de su efectividad. Este "sentido común o sensibilidad neoliberal», provoca que, paulatinamente, quienes nos beneficiamos del sistema, perdamos "la capacidad para detectar lo indetectable, para leer los signos invisibles y para sentir los signos de sufrimiento o de placer del otro" (Berardi, 2017: 11).

En este intercambio de nuestra facultad de sentir por la sensibilidad neoliberal impuesta por el Estado Mórbido, como menciona Valverde, los cuerpos de las mujeres con síndrome de sensibilidad central, "son cuerpos que resuenan, cuerpos que, sin hablar, gritan" (Valverde, 2015: 124). La crítica proviene de esos cuerpos que, sumergidos en la sensibilidad neoliberal, tienen la habilidad para responder en consecuencia; es decir, no solo visibilizar las estructuras perversas del Estado Mórbido, sino generar formas de agenciamiento o, tomando las palabras de Suely Rolnink (2019) «micropolíticas de desestabilización» que permitan la reapropiación de su sensibilidad a partir del saber del cuerpo. La sensibilidad del cuerpo dolorido repolitiza el sufrimiento, lo vuelve carne, lo hace visible. La fibromialgia, nos permite analizar cómo algunos cuerpos, en este caso, los cuerpos doloridos de las mujeres que, durante años condenados al silencio y a la invisibilidad, inesperadamente, se 
muestran y se pronuncian en contra del Estado Mórbido, a partir de aquello a lo que se les condenó en primer lugar: su morbilidad.

\section{BIBLIOGRAFÍA}

Agamben, G. (1995). Homo Sacer. Sovereign Power and Bare Life. Giulio Einaudi editores.

Agamben, G. (1999). Remnants of Auschwitz: The Witness and the Archive. Zone Books.

Agamben, G. (2018). The Sacrament of Language. John Wiley \& Sons.

Ahmed, S. (2004). Affective Economies. Social Text 79, 22(2), 117-139.

Ahmed, S. (2015). La política cultural de las emociones. Programa Universitario de Estudios de Género: Universidad Nacional Autónoma de México

Ahmed, S. (2019b). La promesa de la felicidad. Una crítica cultural al imperativo de la alegría. Caja Negra.

Berlant, L. (2007). Slow Death (Sovereignty, Obesity, Lateral Agency). Critical Inquiry, 33(4), 754-780.

Berlant, L. (2011). Cruel Optimism. Duke University Press.

Emmelhainz, I. (2014). Neoliberalismo y autonomía del arte. Esfera Pública. https://esferapublica.org/nfblog/neoliberalismo-y-autonomia-del-arte/

Emmelhainz, I. (2016). Tiranía del sentido común: La reconversión neoliberal de México. Paradiso.

Foucault, M. (1968). Las palabras y las cosas. Una arqueología de las ciencias humanas (E. C. Frost, Trad.). siglo veintiuno.

Foucault, M. (1998). Historia de la sexualidad I. La Voluntad de Saber (U. Guiñazú, Trad.). Madrid: Siglo XXI editores.

Foucault, M. (2006). Genealogía del racismo. Altamira.

Foucault, M. (2012). Vigilar y Castigar. Biblioteca Nueva.

Foucault, M. (2013). El nacimiento de la clínica. Siglo XXI Editores.

Gómez-Barris, M. (2017). The Extractive Zone: Social Ecologies and Decolonial Perspectives. Duke University Press.

Guattari, F., \& Rolnik, S. (2006). Micropolítica. Cartografías del deseo. Traficantes de Sueños.

Han, B.-C. (2012). La Sociedad del Cansancio (A. Saratxaga Arregi, Trad.). Herder.

López Petit, S. (2009). La movilización global. Breve tratado para atacar la realidad. Traficantes de Sueños. 
López Petit, S. (2014). Hijos de la Luna. Barcelona: Ediciones Bellaterra.

López Petit, S. (2017). La politización del malestar en una sociedad terapéutica. Curso En Tu Interior, Malestar social, (des)politización y psicologías críticashttps://soundcloud.com/traficantesdesue-os/la-politizacion-del-malestar

Lorey, I. (2016). Estado de inseguridad. Gobernar la precariedad. Traficantes de sueños.

Mbembe, A. (2011). Necropolítica (E. Archambault, Trad.). Melusina.

Platero, L. (2014). Las políticas neoliberales contra los derechos sexuales. Fundación Betiko. http://fundacionbetiko.org/wp-content/uploads/2014/02/Politicasneoliberales-contra-los-derechos-sexuales-de-la-ciudadan\%C3\%ADa.pdf

Puar, J. K. (2009). Prognosis time: Towards a geopolitics of affect, debility and capacity, Women \& Performance: a journal of feminist theory, 19:2, 161-172. https://doi.org/10.1080/07407700903034147

Puar, J. K. (2011). Coda: The Cost of Getting Better Suicide, Sensation, Switchpoints. GLQ: A Journal of Lesbian and Gay Studies, 18(1), 150-158. https://doi.org/10.1215/10642684-1422179

Puar, J. K. (2017). The Right to Main. Debility, Capacity, Disability. Duke University Press.

Rolnik, S. (2019). Esferas de la insurrección Apuntes para descolonizar el inconsciente (C. Palmeiro, M. Cabrera, \& D. Kraus, Trads.). Tinta Limón.

Shildrick, M. (2009). Dangerous Discourses of Disability, Subjectivity and Sexuality. Springer.

Shildrick, M. (2015). Living On; Not Getting Better. Feminist Review, 111(1), 10-24. https://doi.org/10.1057/fr.2015.22

Stutzin, V. (2019). Contrapoéticas y fibro-temporalidades del dolor crónico. En M. de Santo (Ed.), La fragilidad del cuerpo amado. Escritos cuir y trans en torno a la politicidad del dolor (pp. 165-219), Barcelona: Continta me tienes.

Sztulwark, D. (2016). Micropolíticas neoliberales, subjetividades de la crisis y amistad política. Micropolíticas neoliberales, subjetividades de la crisis y amistad política // Diego Sztulwark.http://anarquiacoronada.blogspot.com/2016/05/micropoliticasneoliberales.html

Sztulwark, D. (2019, diciembre 30). La sensibilidad es el campo de batalla en este período [Prensa]. https://latinta.com.ar/2019/12/diego-sztulwark-la-sensibilidades-el-campo-de-batalla-en-este-periodo/

Sztulwark, D. (2019, octubre 22). La ofensiva sensible: El síntoma entre el neoliberalismo, el populismo y lo plebeyo // Entrevista a Diego Sztulwark (L. Lewkowicz, S. Lang, L. Feldam, E. Krebs, \& F. Abramovich) [Entrevista]. https://insurgenciamagisterial.com/la-ofensiva-sensible-el-sintoma-entre-elneoliberalismo-el-populismo-y-lo-plebeyo-entrevista-a-diego-sztulwark/ 
Tyner, J. (2019). Dead Labor: Toward a Political Economy of Premature Death. University of Minnesota Press.

Valencia, S. (2010a). Capitalismo Gore. Melusina.

Valencia, S. (2010b). Economía: Cuerpos en negocio. Movimientos en las bases: Transfeminismos, feminismos queer, despatologización, discursos no binarios, Sevilla. http://ayp.unia.es/index.php?option=com_content\&task=view\&id=649

Valverde, C. (2010). «Los cuerpos del delito»: Injusticias y oportunidades en los Síndromes de Sensibilidad Central. PAPELES de relaciones ecosociales y cambio global, 112, 141-153.

Valverde, C. (2012). Malalties-Enfermedades ambientales. https://www.youtube.com/watch?v=xB5D4djbrv0

Valverde, C. (2015). De la necropolítica neoliberal a la empatía radical: Violencia discreta, cuerpos excluidos y repolitización. Icaria.

Valverde, C. (2016). Entrevista a Clara Valverde: "Los espacios de solidaridad fallan por la falta de empatía radical" (H. Rosetti) [Entrevista]. https://kaosenlared.net/entrevista-a-clara-valverde-los-espacios-de-solidaridadfallan-por-la-falta-de-empatia-radical/ 